\title{
Pathogenic properties of enterobacteria isolated from calves in the Far Eastern Federal District
}

\author{
Nikolay Shulga ${ }^{1}$, Natalia Trush $^{2, *}$, Lyudmila Bugaeva ${ }^{1}$, and Irina Sayapina ${ }^{1}$ \\ ${ }^{1}$ Amur State Medical Academy, Blagoveshchensk, Russia \\ ${ }^{2}$ Far Eastern State Agrarian University ,86, Politeknicheskaya Str., Blagoveschensk, Russia
}

\begin{abstract}
Methods of modern control of infectious animal diseases have led to a change in the etiological structure of infectious agents. Irrational use of antibiotic therapy may be the cause of bacterial variability and involvement in the infectious process of opportunistic bacteria that are present in the normal microflora of the animal body. Opportunistic bacteria are the cause of many animal diseases. Infections caused by them have a prolonged character, the pathogenetic basis of which is the persistence of the pathogen in the host body. Bacterial exotoxins damage the plasma membrane of cells. Pore-forming toxins and enzymes disrupt the selective entry and exit of ions through the plasma membrane. This group of toxins includes cytolysins, hemolysins of gram-negative opportunistic enterobacteria, leukotoxins, metalloproteases, and lipases. When pores are formed under the action of hemolysin, secondary processes are triggered that cause the development of pathological consequences. The vaccines and serums produced by biofactories lag behind the practical needs in terms of antigenicity and do not provide protection for newborn calves in the system of anti-epizootic measures. Of particular importance in the etiopathogenesis of acute intestinal disorders is a decrease in colonization resistance of the gastrointestinal mucosa, resulting in an expansion of the spectrum of pathogenic and opportunistic microorganisms that lead to the development of diarrhea.
\end{abstract}

\section{Introduction}

Methods of modern control of infectious animal diseases have led to a change in the etiological structure of infectious agents [1]. Uncontrolled use of antibiotic therapy may be the cause of bacterial variability and the involvement of opportunistic bacteria in the infectious process, which is undoubtedly due to the stimulation of constant variability of bacteria and the involvement of opportunistic microorganisms in the infectious process, which are present as commensals in the normal microflora of the animal body.

Addiction to antibiotics, resistance of pathogenic and opportunistic microorganisms leads to the development of diseases associated with disorders in the intestinal biocenosis. Antibiotics can cause side effects that negatively affect the productivity of animals. The vaccines and serums produced by biofactories lag behind the practical needs in terms of

\footnotetext{
* Corresponding author: Letter_box_n@mail.ru
} 
antigenicity and do not provide protection for newborn calves in the system of antiepizootic measures.

The vaccines and serums produced by biofactories lag behind the established antigenic background in the region and the region. More modern and comprehensive approaches to this issue are needed. It may be worth releasing vaccines and serums from local strains of pathogens.

The manifestation of pathogenic properties of microflora depends on the state of the body and the barrier functions of the mucous membranes and skin. Conditionally pathogenic microorganisms are the cause of many animal diseases [2,3 ]. Infections caused by them tend to have a prolonged course, the pathogenetic basis of which is the persistence of the pathogen in the host $[4,5]$.

Kolibakterioza is one of the most common diseases of young animals. Periodically, this disease is noted in almost all farms of the Amur region. Mass laboratory diagnostics of collibacteriosis of animals began to be carried out in the Amur region in the 60 s of the last century. When studying the collibacteriosis of calves, it was found that the disease outbreaks coincided with the periods of mass calving. V. V. Kolchak (1986) noted that the set of Escherichia serotypes is unstable and changes in each farm. Bacterial exotoxins damage the plasma membrane of cells. Pore-forming toxins and enzymes disrupt the selective entry and exit of ions through the plasma membrane. This group of toxins includes cytolysins, hemolysins of gram-negative opportunistic enterobacteria, leukotoxins, metalloproteases, and lipases. When pores are formed under the action of hemolysin, secondary processes are triggered that cause the development of pathological consequences. In Dalznivi in the mid-90s, a new serum preparation was created - concentrated blood serum (CSC). Its use in cattle breeding helped to reduce the incidence of newborn calves.

Many infections caused by opportunistic microorganisms are considered factorial. Factor infections occur not as a result of relay transmission of the pathogen, but due to microorganisms that constantly live in the animal's body and do not cause disease under normal conditions $[6,7]$.

Conditionally pathogenic microorganisms occupy a significant place in the infectious pathology of animals. [8]. The disease occurs only if animals are exposed to such stressful factors as hypothermia or untimely drinking of colostrum after calving [9].

The sharply continental climate in the Amur region and Khabarovsk territory contributes to the fact that colibacteriosis of calves is widespread, enteropathogenic Escherichia coli is constantly isolated.

Undoubtedly, a decrease in colonization resistance of the gastrointestinal mucosa against the background of irrational use of antibiotics is of particular importance in the etiopathogenesis of acute intestinal disorders, resulting in an increase in the number and range of pathogenic and opportunistic microorganisms, which, in turn, leads to the development of dysbiosis and, as a result, diarrhea [10].

The disease occurs only if the animals are exposed to such stressful factors as hypothermia or untimely milking of colostrum after calving [11, 12, 13]. Undoubtedly, a special significance in the etiopathogenesis of acute intestinal disorders is a decrease in colonization resistance of the gastrointestinal mucosa against the background of irrational use of antibiotics $[14,15]$, which results in an increase in the number and expansion of the spectrum of pathogenic and opportunistic microorganisms [16, 17], which, in turn, leads to the development of dysbiosis and, as a result, diarrhea $[18,19]$.

On the other hand, persistent properties of microorganisms, such as anti - lysozyme, anti-interferon, anti-complementary and DNA-aznaactivity, as well as adhesiveness, are of great importance in the initiation and development of the pathological process [20, 21, 22]. Since these properties increase the resistance of microorganisms to the host's protective 
mechanisms and create certain selective advantages in overcoming the resistance of calves, the study of this phenomenon has become the goal of our research.

\section{Materials and Methods}

The necessary material for the study was selected on dairy farms in the Amur region and Khabarovsk territory. Fecal samples from patients (57 samples) and clinically healthy calves (57 samples) were taken from the rectum with a sterile swab, placed in a sterile saline solution, and immediately delivered for examination. Pure cultures of bacteria were obtained by preparing ten-fold dilutions in a saline solution of the initial suspension, followed by seeding on dense nutrient media. The following media were used in the study: meat-peptone agar, Ploskirev medium, Endo medium, bismuth-sulfite agar, agar with sulfite, salt agar, Chapek medium). The crops were incubated in a thermostat for 24-48 hours at a temperature of $37^{\circ} \mathrm{C}$.

A total of 57 faecal samples from calves with signs of acute intestinal disorders and the same number from clinically healthy calves were selected and analyzed, including 39 samples from calves up to 10 days of age and 18 samples from calves older than 10 days.

Morphological properties of isolated cultures of microorganisms were studied by light microscopy. Identification of isolated pure cultures was performed by morphological, tinctorial, biochemical and serological properties using The "Bergi bacteria Determinant" [5] and "zoopatogenic microorganisms Determinant" [6].

Determination of anti-lysis activity (ALA) of bacteria was performed by photometric method according to the method described earlier by Bukharin O. V. and co-authors [7] and expressed in conventional units (units).

Anti-complementary activity (AKA) was determined by partial hemolysis in gel and expressed in hemolytic units (heme. ed) [8].

Determination of DNA-azic activity was carried out according to the approved method. Deoxyribonucleic acid (DNA, Sigma) was used as a substrate to determine the DNA-azic activity of bacteria. The tested broth cultures were seeded with a loop on the sectors of a Petri dish with meat-peptone agar containing $40 \mathrm{mg}$ of DNA on a stencil. Cups with crops were incubated in a thermostat at $37 \mathrm{oC}$ for 18-20 hours. After the end of incubation, 3-5 ml of $0.1 \mathrm{~N}$ hydrochloric acid solution was applied to the surface of the medium with grown cultures, which was drained after 2 minutes. A transparent zone was formed around the colonies with DNA-azic activity. The width of this zone was measured in $\mathrm{mm}$. The result was expressed as a percentage.

The adhesive properties of microorganisms were determined using native rabbit red blood cells. Red blood cells were previously washed twice with a buffer solution by centrifugation $(300 \mathrm{rpm})$. A $0.1 \mathrm{M}$ solution of sodium phosphate prepared with an isotonic solution of sodium chloride was used as a buffer. To set up the reaction, $0.5 \mathrm{ml}$ of erythrocyte suspension (100 million $/ \mathrm{ml}$ ) was added to $0.5 \mathrm{ml}$ of microbial suspension (109 $\mathrm{CL} / \mathrm{ml}$ ). The mixture was incubated at $37 \mathrm{oC}$ for 30 minutes, then smears were made on slides, fixed with methanol, and stained with methylene blue.

Adhesive properties were assessed by the number of bacteria on the surface of red blood cells. The average adhesion (SPA) was calculated as the average number of microorganisms attached to one erythrocyte, the participation rate of erythrocytes in the adhesive process $(\mathrm{K})$ - as a percentage of erythrocytes having on its surface adhered microbes, the index of adhesiveness of microorganisms (IAM) - the average number of microbial cells on one involved in the adhesive process, the erythrocyte was calculated by the formula: 
The anti-interferon activity of bacteria was studied by an accelerated method [9] and expressed in cu. e. in a Petri dish, $1 \mathrm{ml}$ of daily bacterial culture was uniformly sown on a medium of MPA. Disks containing $\alpha$-interferon produced by FSUE NPO Microgen were placed on the agar surface in concentrations of 5, 2.5, 1.25, 0.62 and $0.32 \mathrm{cu}$. the Cups were placed in a thermostat at $37 \mathrm{oC}$ for 24 hours. About antiinterference activity was judged by the absence of zones of growth inhibition of bacteria around the disks with interferon. The highest concentration of interferon in the disk, around which there is no growth delay zone, was taken as the level of severity of the trait.

Statistical analysis was performed using the method of I. A. Oivin [14]. Intergroup differences were evaluated using the student's t-test. Differences were determined at the level of statistical significance $\mathrm{p}<0.05$.

\section{Results}

Various mechanisms are involved in the pathogenesis of the infectious process. Each type of micro-organism is characterized by a complex of pathogenicity markers.

As a result of the analysis of all the samples obtained, cultures of microorganisms belonging to the following genera were identified: Escherichia, Proteus, Citrobacter, Klebsiella, Enterobacter, Pseudomonas, Morganella, Edwardsiella, Providencia and Hafnia.

Diseases were registered in calves starting from the first day of life and up to 2-3 weeks of growth at all times of the year. But most often in the spring.

An important factor contributing to the survival of microorganisms in the environment and colonization of internal organs is adhesion. Therefore, at the first stage of the study, the adhesive properties of microorganisms were studied for all selected cultures (table 1).

Table 1. Adhesion indicators of isolated microorganisms.

\begin{tabular}{|l|c|c|c|}
\hline \multirow{2}{*}{ Genus of microorganisms } & \multicolumn{3}{|c|}{ Adhesion indicators for the following parameters } \\
\cline { 2 - 4 } & To, \% & SPA & IAM \\
\hline Escherichia $(\mathrm{n}=16)$ & $79.0 \pm 2.5$ & $3.59 \pm 0.41$ & $4.52 \pm 0.31$ \\
\hline Klebsiella $(\mathrm{n}=6)$ & $88.3 \pm 3.2$ & $4.12 \pm 0.52$ & $4.73 \pm 0.42$ \\
\hline Proteus $(\mathrm{n}=13)$ & $89.4 \pm 1.9$ & $4.35 \pm 0.43$ & $4.89 \pm 0.41$ \\
\hline Citrobacter $(\mathrm{n}=9)$ & $84.6 \pm 3.9$ & $3.50 \pm 0.31$ & $4.16 \pm 0.32$ \\
\hline Morganella $(\mathrm{n}=3)$ & $92.3 \pm 2.2$ & $5.50 \pm 0.01$ & $5.75 \pm 0.11$ \\
\hline Edwardsiella $(\mathrm{n}=3)$ & $58.3 \pm 0.1$ & $2.40 \pm 0.01$ & $4.12 \pm 0.12$ \\
\hline Enterobacter $(\mathrm{n}=7)$ & $75.4 \pm 8.1$ & $2.96 \pm 0.81$ & $4.58 \pm 0.60$ \\
\hline Pseudomonas $(\mathrm{n}=3)$ & $97.2 \pm 2.3$ & $4.06 \pm 0.02$ & $4.18 \pm 0.13$ \\
\hline Providencia $(\mathrm{n}=3)$ & $71.1 \pm 0.4$ & $3.00 \pm 0.01$ & $4.20 \pm 0.12$ \\
\hline Hafnia $(\mathrm{n}=3)$ & $54.3 \pm 0.1$ & $2.26 \pm 0.02$ & $4.16 \pm 0.11$ \\
\hline
\end{tabular}

Adhesive activity was evaluated in terms of the index of adhesiveness of microorganisms (IAM), the average adhesion (SPA) and coefficiently of eritrotsitov adhesive process $(\mathrm{K})$. Microbe considered non-adhesive when IAM $\leq 1.75$, nishithini when IAM from 1.76 to 2.50 , sredneamgunsky- from 2.51 to 4.00 and highly adhesive when $\mathrm{IAM} \geq 4.00$. The data presented in Table 1 allow to conclude that all the microorganisms isolated from the intestines of calves, possessed a high degree of adhesiveness, since for all investigated genera of microorganisms indicator IAM was above 4.00. The SPA and K parameters were also equally high for all births.

Many serological types of Escherichia coli were observed in inpatient farms.

Further, other main persistent properties were studied for Escherichia, Proteus, Citrobacter, Klebsiella, Enterobacter, Pseudomonas cultures. The first of the studied properties was anti-complementary activity-AKA. 
The complement system plays an important role in the immune response to microorganisms by modulating the intensity of innate and specific immunity [10]. More than 30 complement proteins are found in serum and other body fluids that form and regulate a cascade of protein transformations that are based on limited proteolysis by endogenous serine proteinases and generate physiologically active substances $(\mathrm{C} 4 \mathrm{a}, \mathrm{C} 3 \mathrm{~A}$, $\mathrm{C} 3 \mathrm{~B}, \mathrm{C} 5 \mathrm{~A}, \mathrm{C} 5 \mathrm{~b}$ ) that determine the effectiveness of the immune system of animals. Complement participates in the formation of adaptive responses of antibody formation to antigens, regulates the size of immune complexes and the degree of their solubility, promotes bacterial lysis, and enhances the action of antibodies.

The results of the study of AKA showed that for cultures of the genera Escherichia, Proteus, Citrobacter and Klebsiella AKA was at a high level and amounted to 40 heme units (table. 2). Cultures belonging to the genera Enterobacter and Pseudomonas did not have anti-complementary activity.

Table 2. Persistent properties of microorganisms.

\begin{tabular}{|l|c|c|c|c|}
\hline \multirow{2}{*}{$\begin{array}{c}\text { Genus of } \\
\text { microorganisms }\end{array}$} & \multicolumn{4}{|c|}{ Persistent properties } \\
\cline { 2 - 5 } & ALA, u. e. & AKA, gem. ed. & AIA, u. e. & DNA-azna, $\%$ \\
\hline Escherichia $(\mathrm{n}=16)$ & $\begin{array}{c}2.15 \pm 0.30 \\
\mathrm{p}<0.05\end{array}$ & $40.0 \pm 0.10$ & $0.39 \pm 0.04$ & $23.1 \pm 0.10$ \\
\hline Proteus $(\mathrm{n}=13)$ & $1.75 \pm 0.34$ & $40.0 \pm 0.09$ & $\begin{array}{c}0.625 \pm 0.02 \\
\mathrm{p}<0,001\end{array}$ & $\begin{array}{c}66.7 \pm 0.80 \\
\mathrm{p}<0,001\end{array}$ \\
\hline Citrobacter $(\mathrm{n}=9)$ & $1.55 \pm 0.25$ & $40.0 \pm 0.08$ & $\begin{array}{c}1.000 \pm 0.10 \\
\mathrm{p}<0.001\end{array}$ & $\sim$ \\
\hline Klebsiella $(\mathrm{n}=6)$ & $2.20 \pm 0.29$ & $40.0 \pm 0.09$ & $\begin{array}{c}0.500 \pm 0.03 \\
\mathrm{p}<0.01\end{array}$ & $\sim$ \\
\hline Enterobacter $(\mathrm{n}=7)$ & $1.15 \pm 0.34$ & $\sim$ & $\sim$ & $\begin{array}{c}50.0 \pm 0.20 \\
\mathrm{p}<0.001\end{array}$ \\
\hline Pseudomonas $(\mathrm{n}=3)$ & $\begin{array}{c}2.65 \pm 0.10 \\
\mathrm{p}<0.001\end{array}$ & $\mathrm{p}>0.05$ & $\sim$ & $\sim$ \\
\hline
\end{tabular}

The reliability of differences was determined by comparing the level of the corresponding parameters with the minimum values of the attribute, $\mathrm{n}$ - the number of isolates. $\sim$ - absence of the parameter.

The study of anti-interferon activity (AIA) of microorganisms showed that representatives of the genera Citrobacter -1.0 u. e. $(p<0.001)$, Proteus -0.625 u.e. ( $p$ $<0.001)$ and Klebsiella -0.5 u.e. $(\mathrm{p}<0.05)$ had a high AIA (table. 2$)$. Low anti - interferon activity was observed for Escherichia cultures-0.39 u.e. for Enterobacter and Pseudomonas cultures, no noticeable AIA was detected. Interferons are proteins whose species-specific action is directed at RNA and DNA - containing viruses, suppressing their replication. In addition, interferon can inhibit cell division, increase the activity of normal killers, and increase phagocytosis [11].

The results of a study of the DNA activity of microorganisms showed that this factor was most pronounced in Enterobacter, Proteus and Escherichia cultures (table 3). For cultures of Citrobacter, Klebsiella and Pseudomonas, DNA-azic activity was not detected.

Table 3. The level of expression of ALA bacteria depending on the source separation.

\begin{tabular}{|l|c|c|c|}
\hline \multirow{2}{*}{$\begin{array}{c}\text { Genus of } \\
\text { microorganisms }\end{array}$} & \multicolumn{2}{|c|}{ The level of ALA for isolates } & \multirow{2}{*}{$\begin{array}{c}\text { The significance of } \\
\text { differences }\end{array}$} \\
\cline { 2 - 3 } & $\begin{array}{c}\text { From clinically healthy } \\
\text { calves }(\mathrm{n}=3)\end{array}$ & $\begin{array}{c}\text { From patients } \\
\text { calves }(\mathrm{n}=3)\end{array}$ & $\mathrm{p}<0.05$ \\
\hline Escherichia & $1.06 \pm 0.14$ & $3.97 \pm 0.40$ & $\mathrm{p}<0.05$ \\
\hline Klebsiella & $1.07 \pm 0.11$ & $3.41 \pm 0.32$ & $\mathrm{p}<0.05$ \\
\hline Proteus & $0.93 \pm 0.19$ & $2.59 \pm 0.30$ & $\mathrm{p}<0.05$ \\
\hline Citrobacter & $1.15 \pm 0.10$ & $1.96 \pm 0.21$ & \\
\hline
\end{tabular}




\begin{tabular}{|l|c|c|c|}
\hline Enterobacter & $1.05 \pm 0.20$ & $1.26 \pm 0.23$ & $\mathrm{p}>0.5$ \\
\hline
\end{tabular}

The reliability of differences was determined when comparing the ALA indicators of healthy and sick calves for the corresponding microorganisms. $\mathrm{n}-\sim$ number of isolates.

And the last of the persistent properties was studied anti-lysozyme (ALA) activity of microorganisms. Lysozyme (synonyms: muramidase, mucopeptidglycohydrolase) is one of the main components of the granular antimicrobial system of neutrophils in animals and humans.

Analysis OF Ala microorganisms showed that the highest activity was in the genus Pseudomonas-2.65 u. e. $(p<0.001)$, followed by Klebscella -2.2 u. e. $(p<0.01)$ and Escherichia -2.15 u. e. $(\mathrm{p}<0.05)$. There was no increase in ALA for representatives of the genera Enterobacter, Citrobacter and Proteus (table 2).

To find out the relationship between the persistent properties of microorganisms and the presence of disease in calves, the level of Ala expression of microorganisms isolated from sick and healthy calves was studied. The results obtained are presented in Table 3.there were no significant differences in ALA for Enterobacter microorganisms for sick and clinically healthy calves.

It should be noted that Enterobacter cultures isolated from calves without clinical manifestations of the disease in more than $40 \%$ of cases did not show persistent properties. However, microorganisms of the genera Proteus, Escherichia, Klebsiella and Citrobacter isolated from sick calves were characterized by a significant Ala level compared to the indicators obtained for isolates isolated from healthy animals. These results suggest that the ALA level of microorganisms of the genera Proteus, Escherichia, Klebsiella and Citrobacter is in direct correlation with the level of pathogenicity of microorganisms for calves.

\section{Discussion}

In the system of antiepizootic measures, one of the factors is the formation of immune livestock. Various mechanisms are involved in the pathogenesis of the infectious process. Each type of micro-organism is characterized by a complex of pathogenicity markers.

When fighting infectious diseases in calves, the difficulty in treatment is not to disrupt the normal microflora, which can prevent or even block the possibility of developing the infectious process. Knowledge of the microbial etiology of the body of calves is essential in the prevention of infectious diseases of various etiologies. Analysis of the microbial landscape of calves ' intestines after weaning indicates that dysbiosis may develop.

In the system of antiepizootic measures, one of the factors is the formation of immune livestock. Various mechanisms are involved in the pathogenesis of the infectious process. Each type of micro-organism is characterized by a complex of pathogenicity markers.

When fighting infectious diseases in calves, the difficulty in treatment is not to disrupt the normal microflora, which can prevent or even block the possibility of developing the infectious process. Knowledge of the microbial etiology of the body of calves is essential in the prevention of infectious diseases of various etiologies. Analysis of the microbial landscape of calves ' intestines after weaning indicates that dysbiosis may develop.

The first moment of the initial stage of interaction of bacteria with the host organism is adhesion, which is carried out in the presence of special adhesins that are part of the surface structures of the cell wall. The microflora that inhabits the body can be a biological barrier, and in stressful situations for the body, a reservoir of infection.

To create passive immunity, antiagesive antitoxic serum is used against Escherichia coliosis of farm animals. 
These results suggest that the level of ALA of microorganisms of the genera Proteus, Escherichia, Klebsiella, and Citrobacter is in direct correlation with the level of pathogenicity of microorganisms for calves.

\section{Conclusions}

The study revealed that diseases of newborn calves in the far Eastern Federal district are caused by many types of pathogenic microorganisms.

Having overcome the protective morpho-physiological capabilities of newborn calves, bacteria synthesize specific toxins in their bodies. Causing the development of certain symptoms of diseases.

These microorganisms are characterized by such virulence factors as a high degree of adhesion, anti-lysozyme, anti-complement and anti-interferon activity, as well as DNA-AZ properties. As a result of the wide spread of these microorganisms, young animals become ill.

The vaccines and serums produced by biofactories lag behind the practical needs in terms of antigenicity and do not provide protection for newborn calves in the system of anti-epizootic measures.

Infections are persistent, and treatment is long and difficult. Our results fully fit into the General theory of microbial variability [12], evidence that due to genetically fixed persistent properties, microorganisms acquire the ability to overcome the protective barriers of the host body's immunity [13].

In the Amur region, during the bacteriological study of pathological material from newborn calves with gastrointestinal disorders, enteropathogenic Escherichia coli has been isolated for many years.

In this regard, there is a need to use local strains of microorganisms (by region) in the production of biologics against diseases of newborn calves of the far Eastern Federal district caused by pathogenic microorganisms.

\section{References}

1. E. Azhmuldinov Comparative assessment of the adaptive ability of various breeds of bulls. Ways to intensify production and processing of agricultural products and current conditions. Materialy mezhdun. scientific and practical, 2 parts, 54-56 (2012)

2. A. Leorda, M. M. Timoshko Prevention of digestive tract dysfunction in calves under transport stress, Veterinary medicine of agricultural animals, 1, 61-63 (2007)

3. L. Teltsov Health and laws of individual development, Materials scientific. practical Conf. dedicated to the memory of prof, 3, 344-358 (2007)

4. L. Teltsov Concept of growing highly productive animals. Materials of the international scientific and practical conference, To the 25th anniversary of the Department of animal science of the Bryansk state agricultural Academy, 21-31 (2008)

5. N. Trush On the relationship between the morphofunctional state of the thyroid and adrenal glands and the safety of calves. International scientific readings: collection of articles of the International scientific and practical conference, 32-36 (2019)

6. V. Lyapina Reducing losses of meat products when weaning calves from cows and subsequent stresses during the period of growing and selling bullocks, 4, 243-246 (2013)

7. Y. Scherbatykh Psychology of stress and methods of correction, 256 (2006) 
8. E. A. Azhmuldinov Comparative assessment of the adaptive ability of various breeds of bulls Materialy mezhdun. scientific and practical, 54-56 (2012)

9. S. Dikunina, L. Plavshak, I. Shulga, N. Krasnojrsk Technological scheme of prevention of respiratory diseases of newborn calves, 12, 198-202 (2015)

10. S. Batanov, S, O. Starostina Milk productivity of first-born cows of different stressresistance, Zootechnia, 2, 18 -19 (2005)

11. E. M. Ueremey Stress state of the body and its influence on the productivity of cows in dairy complexes, Scientific notes of the educational institution "Vitebsk order" Badge of honor " state Academy of veterinary medicine, 2, 143-145 (2011)

12. S. Dikunina, L. Plavshak, I. Shulga, N. Shulga, Technological scheme of prevention of respiratory diseases of newborn calves, 12, 198-202 (20150

13. M. Zurabov, Z. Zurabova Z. Kazan 2012 Some parameters of the adaptation of highly productive cows, imported to the territory of the RT from foreign countries to new conditions, Scientific notes of the Kazan state Academy of veterinary medicine Of N. E. Bauman, 211, 259-262.

14. S. N. Himicheva, L. D. Samusenko Correction of technological stress in young cattle Biology in agriculture, 4 (13), 10 -12 (2016)

15. N. M. Trush Morpho-physiological aspects of growth and adaptation, International scientific readings: collection of articles of the International scientific and practical conference, 11-14 (2020)

16. N. M. Zimin,Hormones and their role in metabolism, Veterinary medicine of agricultural animals, 9, 4-11 (2006)

17. L. Karpenko, A. Enukashvili Yu. Seasonal changes in the mineral composition of blood serum of cattle, Materials of H1P mezhdunarod. Moscow veterinary Congress, $201(2005)$

18. V. Levakhin, E. Azhmuldinov, M. Titov Growth Intensity and adaptive qualities of bulls of various breeds under the influence of technological stress factors. Bulletin of meat cattle breeding, 31(89), 54-58 (2015)

19. I. Lyapina, E. Azhmuldinov, N. Belov Morphological and biochemical parameters of blood of young cattle under various conditions of its maintenance, News of the Oreburg state agrarian University, 136-138 (2006)

20. 20. N. Shulga, D. Petrukhin, D. Zhelyabovskaya Some aspects of colostral immunity formation in newborn animals, 8, 136-139 (2012)

21. A. Salo, V. Popov, M. Poberukhin, Stress Tolerance of purebred and crossbred young animals to transport and pre-slaughter stress, Innovative ways to improve the efficiency of agricultural production mater. International. science-practice, 116-117 (2010)

22. A. Konovalov Methodology and practice of assessing the stress resistance of cattle, Vestnik agro-industrial complex of the upper Volga region, 1, 38 -42 (2011) 